\title{
Don't blame the nails for SARS-CoV-2 hospitalizations and mortality
}

\author{
Amar D. Desai ${ }^{1} \cdot$ Rhiannon C. Miller $^{2} \cdot$ Shari R. Lipner ${ }^{2}$ (I) \\ Received: 16 January 2022 / Revised: 16 January 2022 / Accepted: 10 February 2022 / Published online: 17 February 2022 \\ (c) The Author(s), under exclusive licence to Springer-Verlag GmbH Germany, part of Springer Nature 2022
}

Keywords Onychomycosis $\cdot$ SARS-CoV-2 $\cdot$ COVID-19 $\cdot$ Nail infection $\cdot$ Hospitalization

\section{To the editor}

We read with interest Rakita et al.'s retrospective cohort study investigating SARS-CoV-2 severity in adult outpatients and inpatients with onychomycosis and other skin diseases [1]. The authors reported that $23 \%$ of SARSCoV-2-positive patients with a dermatologic condition had onychomycosis. COVID-19 patients with onychomycosis vs. COVID-19 patients with a different dermatologic condition were more likely to be hospitalized, seek in-patient care, require oxygen therapy, and have severe-critical COVID-19 with a higher mortality rate. In this letter, we analyze the methodology used by Rakita et al., and review the literature on prevalence of onychomycosis and long-term outcomes.

Rakita et al. reported that nearly $25 \%$ of SARS-CoV-2 patients with dermatologic conditions had onychomycosis. A review of 21 North American hospitals found a hospital prevalence of $8.9 \%$ (95\% CI 4.3-13.6\%) and a population prevalence of $4.3 \%$ (95\% CI 1.9-6.8) for onychomycosis after excluding studies with diagnosis based on clinical examination only [2]. Therefore, the remarkably high rate reported by Rakita et al. is likely due to problematic methodology with onychomycosis diagnosis, with patients "diagnosed clinically by a dermatologist and confirmed with culture analysis as needed". The diagnosis of onychomycosis cannot be made using clinical examination alone. It requires mycological confirmation [3]. Therefore, many of

This comment refers to the article available online at https://doi. org/10.1007/s00403-021-02299-8.

Shari R. Lipner

sh19032@med.cornell.edu

1 Rutgers New Jersey Medical School, Newark, NJ, USA

2 Department of Dermatology, Weill Cornell Medicine, 1305 York Avenue, 9th Floor, New York, NY 10012, USA the patients in Rakita et al.'s study likely also had other nail diseases, other than onychomycosis.

The authors also report several binary logistic regression models for COVID-19 outcomes, with onychomycosis vs. non-onychomycosis patients being more likely to be hospitalized (OR 3.56). Diabetes mellitus, peripheral vascular disease, and immunosuppression may increase predisposition to onychomycosis, with poorer prognosis for treatment, but there is no evidence to suggest that these patients are more likely to be hospitalized due to their nail disease alone [4]. Rakita et al. showed that on multinomial logistic regression, controlling for a number of demographic/comorbidity variables, that onychomycosis does not impact hospital duration, COVID-19 course (recovered vs. chronic complications), or death. It is unclear why the authors chose to assess outcomes in a binomial fashion and only hospitalization and disease course in a multinomial fashion.

After controlling for demographic variables, including age, sex, and race, as well as, co-morbidities including AIDS, diabetes, and cancer, there was no longer an association between hospitalization duration, in-patient visits, COVID-19 severity, or mortality rate, suggesting that there are confounding factors. While rate of hospitalization remained significant, hospitalized patients are much more likely to have a full examination, including skin and nails, than patients in emergency rooms and outpatient settings. Furthermore, a number of confounders associated with COVID-19-related mortality, such as COPD and hypertension, were omitted [5].

In sum, we highlight the importance of understanding the effects of co-morbidities associated with COVID-19 infection when drawing conclusions regarding dermatologic conditions, including onychomycosis, which has similar comorbidities and is not independently associated with increased COVID-19 severity or mortality. We stress the importance of considering broad nail pathology differentials beyond onychomycosis and performing mycological testing. Additional 
research is required to further understand whether dermatologic-specific conditions, including onychomycosis have an effect on outcomes and disease course following COVID-19 infection.

\section{Funding None.}

\section{Declarations}

Conflict of interest The authors declared that they have no conflict of interest.

\section{References}

1. Rakita U et al (2021) Associations between onychomycosis and COVID-19 clinical outcomes: a retrospective cohort study from a US metropolitan center. Arch Dermatol Res. https://doi.org/10. 1007/s00403-021-02299-8

2. Sigurgeirsson B, Baran R (2014) The prevalence of onychomycosis in the global population: a literature study. J Eur Acad Dermatol Venereol 28:1480-1491

3. Lipner SR, Scher RK (2016) Onychomycosis-a small step for quality of care. Curr Med Res Opin 32:865-867

4. Lipner SR, Scher RK (2019) Onychomycosis: treatment and prevention of recurrence. J Am Acad Dermatol 80:853-867

5. Parker JJ et al (2021) Characteristics, comorbidities, and data gaps for coronavirus disease deaths, Tennessee, USA. Emerg Infect Dis $27: 2521-2528$

Publisher's Note Springer Nature remains neutral with regard to jurisdictional claims in published maps and institutional affiliations. 